PROCEEDINGS OF THE

AMERICAN MATHEMATICAL SOCIETY

Volume 127, Number 5, Pages 1265-1267

S 0002-9939(99)04634-1

Article electronically published on January 27, 1999

\title{
THE IDEAL OF POLYNOMIALS VANISHING ON A COMMUTATIVE RING
}

\author{
ROBERT GILMER
}

(Communicated by Wolmer V. Vasconcelos)

\begin{abstract}
We determine equivalent conditions on a commutative Artinian ring $S$ in order that the ideal of $S[t]$ consisting of polynomials that vanish on $S$ should be principal. Our results correct an error in a paper of Niven and Warren.
\end{abstract}

Let $R$ be a commutative unitary ring. If $f(t)=\sum_{j=0}^{n} a_{j} t^{j} \in R[t]$, then $f$ induces a polynomial function $T_{f}$ of $R$ into $R$ defined by $T_{f}(r)=\sum_{j=0}^{n} a_{j} r^{j}$. The map $f \rightarrow T_{f}$ is a surjective homomorphism of $R[t]$ onto the ring $\mathcal{P}(R)$ of all polynomial functions of $R$ into $R$. Following Narkiewicz [N, p. 1], we denote by $I_{R}$ the kernel of this map. Thus $I_{R}=\left\{f \in R[t] \mid T_{f}=0\right\} ; I_{R}$ is called the ideal of polynomials that vanish on $R$. In [NW], Niven and Warren determine a set of generators for $I_{R}$ in the case where $R=\mathbb{Z} / m \mathbb{Z}$ is the ring of integers modulo $m$, and for this ring they use the notation $\mathcal{I}(m)$ instead of $I_{R}$. Exercise 1, page 10, of [N] states that $\mathcal{I}(m)$ is principal if and only if $m$ is prime; this repeats the content of Theorem 4 of $[\mathrm{NW}]$. However, that result is false, with the correct statement being that $\mathcal{I}(m)$ is principal if and only if $m$ is square-free. This note corrects the error in [NW] by showing that, for a finite ring $R$, the ideal $I_{R}$ is principal if and only if $R$ is reduced or, equivalently, if and only if $R$ is a direct sum of fields. We begin with a basic lemma.

Lemma 1. If $e$ is an idempotent of the commutative unitary ring $R$, the epimorphism $\phi: R[x] \rightarrow R e[x]$ defined by $\phi(f(x))=e f(x)$ maps $I_{R}$ onto $I_{R e}$.

Proof. The inclusion $\phi\left(I_{R}\right) \subseteq I_{R e}$ is clear. To prove the converse we show that $I_{R e} \subseteq I_{R}$; this suffices since $\phi$ induces the identity map on $R e[x]$. Thus, take $g \in I_{R e}$. Since $g(0)=0, g=e x \cdot h$ for some $h \in R e[x]$, and hence $g$ vanishes on $R(1-e)$. Because $g$ vanishes on $R e$ and $R=R e \oplus R(1-e)$, it follows that $g \in I_{R}$.

Corollary 2. If $R=R_{1} \oplus \ldots \oplus R_{n}$ is the direct sum of ideals $R_{1}, \ldots, R_{n}$ of $R$, then $R[x]=\sum_{j=1}^{n} \oplus R_{j}[x]$ and $I_{R}=\sum_{j=1}^{n} \oplus I_{R_{j}}$. Therefore $I_{R}$ is principal as an ideal of $R[x]$ if and only if each $I_{R_{j}}$ is principal as an ideal of $R_{j}[x]$.

If $S$ is an Artinian ring, it is well-known that $S$ is a finite direct sum of zerodimensional local rings [ZS, Theorem 3, p. 205]. Hence Corollary 2 shows that in

Received by the editors June 10, 1997 and, in revised form, August 6, 1997.

1991 Mathematics Subject Classification. Primary 13B25; Secondary 13E10.

Key words and phrases. Vanishing polynomials, Artinian rings.

(C)1999 American Mathematical Society 
determining conditions under which $I_{S}$ is principal, it suffices to consider the case where $S$ is local. Our solution of this problem in Corollary 5 uses the following result due to Ernst Snapper.

Theorem 3 (Snapper [S, p. 680]). Suppose $R$ is a commutative unitary ring and $f(t) \in R[t]$ is not a zero divisor in $R[t]$. If $d$ is the minimum of the degrees of the nonzero elements of the principal ideal $(f(t))$ of $R[t]$, then there exists $a \in R$ such that af $(t)$ has degree $d$.

Theorem 4. If $(R, M)$ is a zero-dimensional local ring, then $I_{R}$ is principal if and only if either $R / M$ is infinite or $R$ is a finite field.

Proof. If $R$ is a finite field with $q$ elements, it is well-known that $I_{R}=\left(t^{q}-t\right)$. If $R / M$ is infinite, we show that $I_{R}=(0)$ (cf. [J, Theorem 9]). Thus, let $f(t)=$ $\sum_{j=0}^{n} f_{j} t^{j} \in I_{R}$ and choose elements $a_{1}, a_{2}, \ldots, a_{n+1}$ in distinct residue classes of $M$ in $R$. Since $f\left(a_{1}\right)=0, f(t)$ is divisible by $\left(t-a_{1}\right)$ in $R[t]$. For $1 \leq k<n+1$, if $f(t)$ is divisible by $\left(t-a_{1}\right) \ldots\left(t-a_{k}\right)$ in $R[t]$, say $f(t)=\left(t-a_{1}\right) \ldots\left(t-a_{k}\right) g(t)$, then $0=f\left(a_{k+1}\right)=\left(a_{k+1}-a_{1}\right) \ldots\left(a_{k+1}-a_{k}\right) g\left(a_{k+1}\right)$, where each $a_{k+1}-a_{i}$ is a unit of $R$. We conclude that $g\left(a_{k+1}\right)=0, g(t)$ is divisible by $t-a_{k+1}$, and hence $f(t)$ is divisible by $\left(t-a_{1}\right) \ldots\left(t-a_{k+1}\right)$ in $R[t]$. By induction it follows that $f(t)$ is divisible by $\left(t-a_{1}\right) \ldots\left(t-a_{n+1}\right)$, and hence $f(t)=0$. Thus $I_{R}=(0)$ if $R / M$ is infinite.

To prove the converse it suffices to show that $I_{R}$ is not principal if $R / M$ is finite and $M \neq(0)$. We use a proof by contradiction. Assume $I_{R}=(g(t))$, let $q=|R / M|$, and choose $e>1$ so that $(0)=M^{e}<M$. Since $\left(t^{q}-t\right)^{e} \in I_{R}$, the polynomial $g(t)$ has a unit coefficient. If $b$ is a nonzero element of $\operatorname{Ann}(M)$, then $b\left(t^{q}-t\right) \in I_{R}$, and the proof in the preceding paragraph shows that $I_{R}$ contains no nonzero element of degree less than $q$. Hence Theorem 3 shows that $a g(t)=\sum_{i=0}^{q} c_{i} t^{i}$ has degree $q$ for some $a \in R$. We show that each $c_{i}$ belongs to $\operatorname{Ann}(M)$. Thus, let $u_{0}$ be an arbitrary element of $M$ and choose $u_{1}=0, u_{2}, \ldots, u_{q}$ to be a set of representatives of the residue classes of $M$ in $R$. Viewing $c_{0}, c_{1}, \ldots, c_{q}$ as a solution in $R$ of the homogenous system

$$
\sum_{j=0}^{q} x_{j} u_{i}^{j}=0, \quad 0 \leq i \leq q
$$

of equations, it follows that $c_{j} d=0$ for $0 \leq j \leq q$, where $d=\prod_{i<j}\left(u_{i}-u_{j}\right)$ is the Vandermonde determinant associated with $u_{0}, u_{1}, \ldots, u_{q}$. Since $d$ is a unit multiple of $u_{0}$, it follows that $c_{j} u_{0}=0$ for each $j$, and hence each $c_{j}$ is in $\operatorname{Ann}(M)$, as asserted. Because $g$ has a unit coefficient, $a$ is also in $\operatorname{Ann}(M)$. Now $a g-c_{q}\left(t^{q}-t\right) \in I_{R}$, and because $I_{R}$ contains no nonzero polynomial of degree less than $q, a g=c_{q}\left(t^{q}-t\right)$. We conclude that exactly two of the coefficients of $g$ are units - those of $t^{q}$ and of $t$. Moreover, since $g(0)=0$, we have $g(t)=u t^{q}+v t+t^{2} h(t)$ for some units $u, v$ of $R$ and polynomial $h(t) \in R[t]$. Thus $g(a)=v a \neq 0$, a contradiction to the fact that $g(t) \in I_{R}$. Therefore $I_{R}$ is not principal, as asserted.

Since a zero-dimensional local ring $(R, M)$ is finite if and only if $R / M$ is finite, part(a) of Corollary 5 is a consequence of Theorem 4.

Corollary 5. Let $S$ be an Artinian ring.

(a) $I_{S}$ is principal if and only if $S$ is a direct sum of finite fields and of infinite zero-dimensional local rings. 
(b) If $S$ is finite, then $I_{S}$ is principal if and only if $S$ is reduced or, equivalently, if and only if $S$ is a direct sum of finite fields.

\section{REFERENCES}

[J] G. Jacob, Anneau de fonctions polynomes d'un anneau commutatif unitaire, Commun. Algebra 8 (1990), 793-811. MR 82j:13007

[N] W. Narkiewicz, Polynomial Mappings, Lecture Notes in Math. 1600 (1995). MR 97e:11037

[NW] I. Niven and D. Warren, A generalization of Fermat's Theorem, Proc. Amer. Math. Soc. 8 (1957), 306-313.

[S] E. Snapper, Completely primary rings I., Annals of Math. 52 (1950), 666-693. MR 12:314b

[ZS] O. Zariski and P. Samuel, Commutative Algebra, vol. I, Springer, Berlin-Heidelberg, 1986.

Department of Mathematics, Florida State University, Tallahassee, Florida 323064510

E-mail address: gilmer@math.fsu.edu 